\title{
Fuzzy modeling, maximum likelihood estimation, and Kalman filtering for target tracking in NLOS scenarios
}

\author{
Jun $\mathrm{Yan}^{1^{*}}$, Kegen $\mathrm{Yu}^{2}$ and Lenan $\mathrm{Wu}^{3}$
}

\begin{abstract}
To mitigate the non-line-of-sight (NLOS) effect, a three-step positioning approach is proposed in this article for target tracking. The possibility of each distance measurement under line-of-sight condition is first obtained by applying the truncated triangular probability-possibility transformation associated with fuzzy modeling. Based on the calculated possibilities, the measurements are utilized to obtain intermediate position estimates using the maximum likelihood estimation (MLE), according to identified measurement condition. These intermediate position estimates are then filtered using a linear Kalman filter (KF) to produce the final target position estimates. The target motion information and statistical characteristics of the MLE results are employed in updating the KF parameters. The KF position prediction is exploited for MLE parameter initialization and distance measurement selection. Simulation results demonstrate that the proposed approach outperforms the existing algorithms in the presence of unknown NLOS propagation conditions and achieves a performance close to that when propagation conditions are perfectly known.
\end{abstract}

Keywords: Fuzzy modeling; Probability-possibility transformation; Non-line-of-sight; Maximum likelihood estimator; Kalman filter; Target tracking

\section{Introduction}

Wireless positioning has received considerable attention in recent years [1,2]. The provision of accurate mobile position information has become increasingly important since regulations for emergency services and commercial application for location-based services (LBS) were issued by the US Federal Communications Commission (FCC) and other government bodies. Reliable location estimation is a critical component for a wide range of applications including medical services, search and rescue operations, and intelligent transport system (ITS). In order to enhance the performance of positioning systems or networks, effective advanced position estimation techniques are required especially for scenarios where radio propagation is complex.

In the presence of non-line-of-sight (NLOS) radio propagation, the angle, time of arrival (TOA), and distance

\footnotetext{
* Correspondence: yanj@njupt.edu.cn

${ }^{1}$ College of Telecommunications and Information Engineering, Nanjing University of Posts and Telecommunications, Nanjing 210003, China Full list of author information is available at the end of the article
}

measurement error consist of a noise component and a bias component, both of which are random [2]. NLOS propagation also makes it difficult to model accurately the received signal power. Thus, NLOS propagation poses a great challenge for achieving accurate position estimation. In the literature, a variety of methods and techniques have been proposed to mitigate NLOS propagation effect. A database of field measurements (e.g., received signal strength) at fixed locations in a specific propagation environment can be utilized to improve position accuracy $[3,4]$. Because the cost for database creation and maintenance is high, it is impractical to employ such an approach for many applications. In the case of single-shot positioning, optimization is typically employed to minimize a cost function which includes the bias component [5-8]. When prior statistical knowledge of the NLOS error is unknown, line-of-sight (LOS) and NLOS identification can be used to mitigate the distance bias error using online mean and variance estimates. The velocity and heading angle information 
as well as the smoothed distance measurements can be incorporated through the extended Kalman filter (KF) to determine the mobile position [9]. However, the NLOS identification is an exhausting process, since a successive sequence to measurements is employed for the identification purpose. In addition, it is not suitable for real-time tracking because of the time delay involved. In the case of known noise and bias statistics, statistical based NLOS mitigation algorithm can be used to achieve highly accurate position estimates $[10,11]$. In order to improve the NLOS identification performance under the worst condition, it requires additional hardware utilization (antenna array) or higher computational complexity (deletion diagnostics scheme) [10]. Moreover, this method can only work well in the presence of a large number of BSs, among which only a small number of BSs are NLOS BSs. In the event that the target is moving, geometry and motion characteristics are usually utilized to reduce the NLOS effect such as through Kalman filtering [12-14]. For motion information utilization in [14], some additional hardware is required, making it difficult to implement in practice. Based on the measurement data selection over a period of time, an LOS-only-based approach for positioning in NLOS environments was proposed in [15]. The measurements at NLOS BSs are excluded, so the potential accuracy gain using NLOS measurements is lost. Considering the LOS/NLOS transition as a Markov process, a number of mobile tracking algorithms were proposed in [16-18]. The limitation is its higher system complexity, since a bank of filters (LOS and NLOS modes) is used. Based on the accurate model of mobile motion and information from location measurements at different time instances, a model-based dynamic filter was proposed to improve the location estimation in [19]. However, such a method requires the knowledge of the parameters of mobile terminal motion model and the control input decision model in advance. A joint particle filter and UKF position tracking in severe NLOS situations was proposed in [20]. Because of the higher computational complexity, it may be inappropriate to apply this method to complexity-constrained scenarios.

In this paper, we focus on the maximum likelihood estimation (MLE)-based position estimation algorithm. Although extensive investigations have been conducted for positioning in NLOS scenarios, the unknown LOS and NLOS conditions make it difficult directly to apply the MLE for position determination. In addition, since MLE makes use of nonlinear optimization in the presence of a nonlinear likelihood function such as in radio positioning, parameter initialization is required, which can be a difficult issue. Thus, the development of suitable techniques is required to enable the MLE to achieve superior position estimation performance.
Motivated by the above discussions, we propose a new three-step approach for target tracking, which is simple yet effective. First, we propose to use a fuzzy model to deal with measurement condition estimation. The probabilitypossibility transformation is utilized to calculate the possibility that a measurement is corrupted only by noise. Whether or not a measurement is selected is based on the quality of the measurement or on the value of the possibility. Second, KF design is performed to incorporate the covariance matrix of MLE and the target motion information. Using the position prediction from the KF, the problem of parameter initialization for optimization can be readily resolved.

The main contributions of the paper are summarized as follows:

1. The aim of the fuzzy modeling proposed in [21] was to use the sensor measurement data for derivation and fusion; however, in this paper, the fuzzy modeling is introduced for measurement condition estimation. Thus, a new application scenario of fuzzy modeling is proposed in this paper.

2. The measurement data selection in [15] relies on the measurement record over a significant long period of time, and the predefined number of the LOS measurement is also difficult to set for different environments, so this kind of method is unrealistic for many real-time application scenarios. Meanwhile, because only the LOS measurements were selected for final position estimation, some performance gain from using the NLOS measurements will be lost. However, in this paper, the measurement selection only requires the current distance measurement. Therefore, the proposed measurement selection approach has some advantages in time cost and system complexity for diverse channel environments. In addition, the NLOS measurements are also utilized for final position estimation, and the proposed algorithm performs better than the method in [15].

3. Although the author of [11] proposed the MLE for mobile location estimation, it is not suitable for practical application because of the need of complex LOS/NLOS identification. In the proposed MLE, owing to the Kalman position prediction feedback and fuzzy modeling, it is easier to realize the LOS/ NLOS identification, making it a more practicable approach.

4. In this paper, different from the KF technique for mobile location estimation in [9], a KF is utilized to refine the position estimation after the MLE. Also, the KF prediction is used for fuzzy modeling construction and can help the NLOS identification. Thus, the utilization of KF is more effective. 
The remainder of this paper is organized as follows. The distance measurement model in LOS and NLOS conditions and the distribution of the measurement noise and bias error are given in Section 2. The proposed three-step approach is described in detail in Section 3. Simulation and results are reported in Section 4. Finally, Section 5 draws our conclusion.

\section{Measurement model}

Consider mobile tracking by use of distance measurements in a cellular network or a wireless sensor network. Let $\left(x_{k}, y_{k}\right)$ and $\left(x_{\mathrm{BS}_{i}}, y_{\mathrm{BS}_{i}}\right)$ denote the two-dimensional positions of the mobile and the $i$ th base station (BS) at time instant $k$, respectively. Then, the Euclidian distance between them is given by

$$
d_{i, k} \triangleq \sqrt{\left(x_{k}-x_{\mathrm{BS}}\right)^{2}+\left(y_{k}-y_{\mathrm{BS}}\right)^{2}}
$$

where $i \in\{1,2, \ldots N\}$ and $N$ is the BS population. In LOS condition, $d_{i, k}$ is only corrupted by measurement noise $n_{i, k}$, which can be modeled as a zero-mean white Gaussian variable $N\left(0, \sigma_{i}^{2}\right)$. However, in NLOS condition, $d_{i, k}$ is corrupted by both measurement noise $n_{i, k}$ and NLOS bias error $e_{i, k}$. The measurement equations can be simply written as follows:

$$
\begin{aligned}
& \text { LOS condition, } z_{i, k}=d_{i, k}+n_{i, k} \\
& \text { NLOS condition, } z_{i, k}=d_{i, k}+n_{i, k}+e_{i, k}
\end{aligned}
$$

In the literature, the bias error is modeled as a random variable which has an exponential or Gaussian distribution. In this paper, the bias error is assumed to have either a Gaussian distribution $N\left(\mu, \sigma_{\mathrm{NLOS}}^{2}\right)$ [22,23] or an exponential distribution $e(\lambda)$ (mean $=\lambda$ and variance $=\lambda^{2}$ ) [5]. Given the distance measurements and the knowledge of the noise and bias error statistics, the question is how to reliably and accurately track the target position through mitigating the NLOS effect. In the next section, a new approach is proposed to achieve the goal.

\section{Proposed approach}

The block diagram of the proposed three-step approach is illustrated in Figure 1, which contains three main steps: (1) fuzzy modeling of each measurement data, (2) MLE-based single-shot position determination, and (3) KF-based position filtering. First, the possibility that a measurement is only corrupted by noise is calculated according to the probability-possibility transformation model. The measurements with high possibility and low possibility are considered as the LOS and NLOS measurements for MLE-based position calculation, respectively. Second, the intermediate single-shot position estimates are obtained using the MLE. At last, KF is utilized to produce the smoothed position estimates. In this step, target motion information and statistical characteristics of MLE are employed for performance improvement. The details of the three main steps are described in Figure 1.

\subsection{Fuzzy modeling for measurement selection}

The proposed fuzzy modeling for measurement condition estimation contains three steps as shown in Figure 2: (1) fuzzy model parameter preparation, (2) probabilitypossibility transformation, and (3) measurement condition estimation. Probability theory and possibility theory are the two main methods to describe the measurement uncertainty. In the probability theory, the measurement uncertainty is described by a probability distribution based on the available statistics. But such a method has a higher computational complexity. In order to perform effectively in the scenarios with limited computation capabilities, possibility theory prefers to adopt. In this paper, the basic possibility theory for fuzzy modeling of measurement data originally developed in [21] is adopted. This method avoids the complex associated computations and the further treatment of the propagation of information is easier to realize, especially because of the simple parameterized shape of possibility distribution. In this theory, a truncated triangular probability-possibility transformation is utilized to

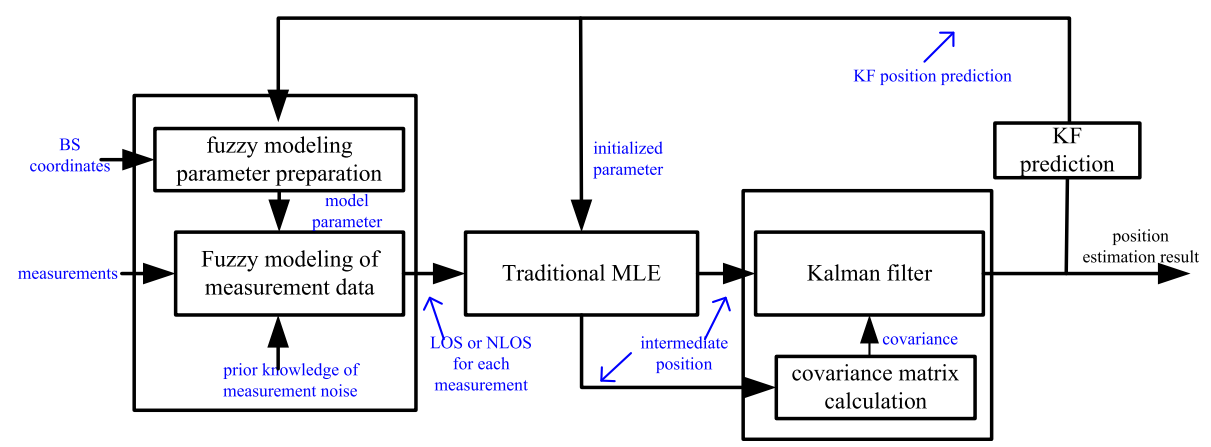

Figure 1 Block diagram of the proposed three-step positioning and tracking algorithm. 


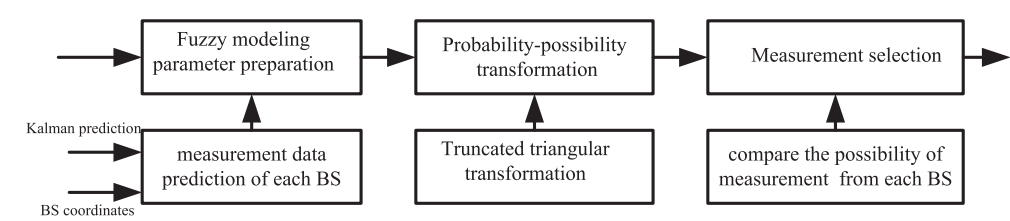

Figure 2 Block diagram of fuzzy modeling for measurement selection.

describe the possibility of a specific type of measurement from a physical sensor. Applying this theory, fuzzy modeling of measurements associated with each BS is established to calculate the possibility that the corresponding measurement is taken under LOS propagation. To perform such a transformation, some prior information is required and it can be considered as an approximation of the optimal transformation.

The relationship between the probability and possibility is established through a probability-possibility transformation. As shown in Figure 3, the truncated triangular probability-possibility transformation is described by the following:

$$
\pi(x)=\left\{\begin{array}{cc}
1-(1-\varepsilon)\left|x-x_{c}\right| /\left(x_{\varepsilon}-x_{c}\right) & i f\left|x-x_{c}\right| \leq\left(x_{\varepsilon}-x_{c}\right) \\
\varepsilon & i f\left(x_{\varepsilon}-x_{c}\right) \leq\left|x-x_{c}\right| \leq\left(x_{n}-x_{c}\right) \\
0 & i f\left|x-x_{c}\right| \geq\left(x_{n}-x_{c}\right)
\end{array}\right.
$$

The determination of the parameters such as $x_{c}, x_{n}$, $x_{\varepsilon}$, and $\varepsilon$ can be found in Table 1 for different measurement noise law [21]. $x_{m}$ and $\sigma$ represent the mean and the standard deviation of the measurement data, respectively. In this paper, the measurement noise follows the Gaussian law, thus, $x_{c}$ equals to $x_{m}$. Moreover, the acquirement of the mean is realized using the Kalman

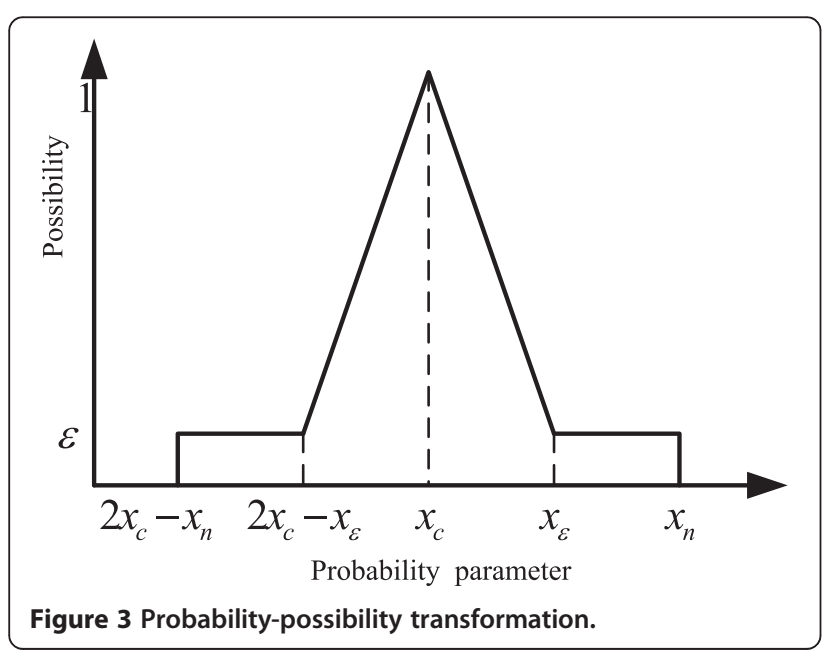

position prediction. For a given BS, the mean for the transformation can be calculated by

$$
x_{m}=m=\sqrt{\left(x_{k / k-1}-x_{\mathrm{BS}_{i}}\right)^{2}+\left(y_{k / k-1}-y_{\mathrm{BS}_{i}}\right)^{2}}
$$

After the probability-possibility transformation, each measurement is assigned with a specific possibility associated with the LOS propagation. A measurement with a high possibility is likely a measurement under LOS condition. Furthermore, a measurement with a low possibility is likely a measurement under NLOS condition. In this paper, we investigate two different methods for determining the number of LOS measurements selected for position determination. One is that the number is predefined and fixed, whereas the other is that any measurement whose probability is greater than a predefined value is selected. Clearly, the number in the second case is variable.

Note that there are two probabilities associated with fuzzy modeling. One is the false alarm probability $P_{\mathrm{FA}}$ which considers the measurement in LOS scenario as NLOS measurement, while the other is the detection probability $P_{\mathrm{D}}$ which considers the measurement in NLOS scenario as NLOS measurement.

Assuming NLOS error with a Gaussian distribution $N\left(\mu, \sigma_{\mathrm{NLOS}}^{2}\right)$, for a given BS, let us denote the error-free TOA measurement as $m$. Then, the two probability functions under LOS and NLOS conditions are shown in Figure 4. Taking Neyman-Pearson (NP) detection theory as a parameter selection criterion, in which the minimum $P_{\mathrm{FA}}$ is chosen as the given false alarm probability, we can obtain that the left endpoint and right endpoint of the given false alarm probability are $m$ $2.58 \sigma_{i}$ and $m+2.58 \sigma_{i}$, respectively, and the predefined

Table 1 The parameters of the probability-possibility transformation

\begin{tabular}{lcccc}
\hline & $\boldsymbol{x}_{\boldsymbol{c}}$ & $\boldsymbol{x}_{\boldsymbol{n}}$ & $\boldsymbol{x}_{\boldsymbol{\varepsilon}}$ & $\boldsymbol{\varepsilon}$ \\
\hline Gaussian law & $x_{m}$ & $x_{m}+2.58 \sigma$ & $x_{m}+1.54 \sigma$ & 0.12 \\
Exponent law & $x_{m}$ & $x_{m}+3.2 \sigma$ & $x_{m}+1.46 \sigma$ & 0.13 \\
Triangular law & $x_{m}$ & $x_{m}+2.45 \sigma$ & $x_{m}+1.63 \sigma$ & 0.11 \\
Uniform law & $x_{m}$ & $x_{m}+1.73 \sigma$ & $x_{m}+1.73 \sigma$ & 0 \\
\hline
\end{tabular}




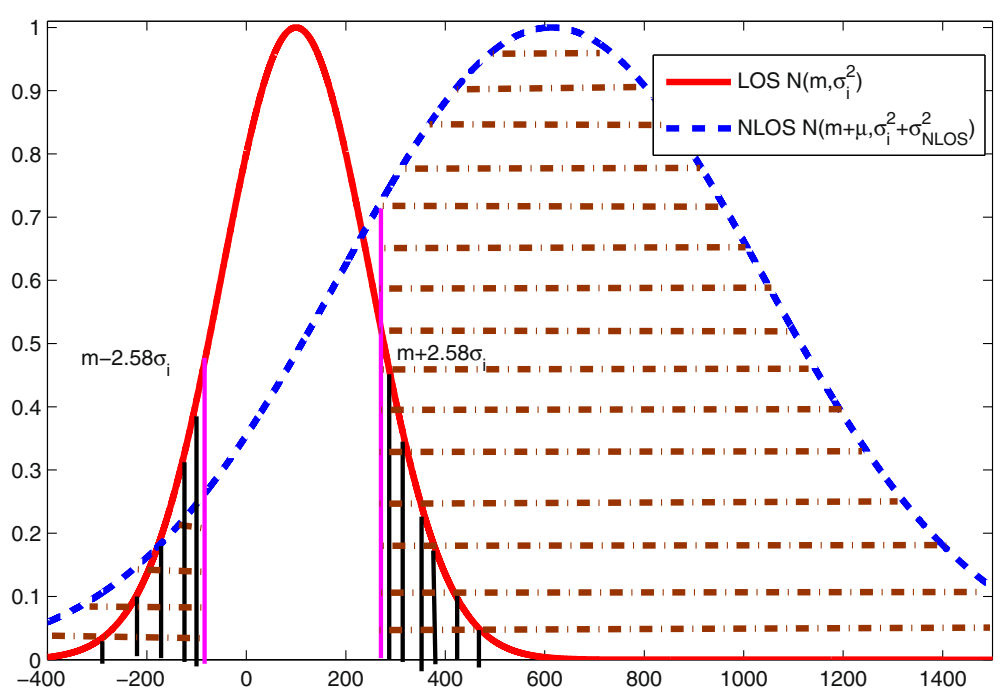

Figure 4 Calculation of loss probability and error probability.

possibility parameter $\varepsilon=0.12$ (see Appendix for more details).

Since the distribution of the measurement assumed in LOS condition can be described as $N\left(m, \sigma_{i}^{2}\right)$, the false alarm probability $P_{\mathrm{FA}}$ is obtained by

$$
\begin{aligned}
P_{\mathrm{FA}} & =\Phi\left(\frac{m-2.58 \sigma_{i}-m}{\sigma_{i}}\right)+\left(1-\Phi\left(\frac{m+2.58 \sigma_{i}-m}{\sigma_{i}}\right)\right) \\
& =2(1-\Phi(2.58))=2(1-0.995)=0.01
\end{aligned}
$$

where

$$
\Phi(\lambda)=\frac{1}{\sqrt{2 \pi}} \int_{-\infty}^{\lambda} e^{\frac{-x^{2}}{2}} d x
$$

Similarly, the distribution of the measurement assumed in NLOS condition can be defined as $N$ $\left(m+\mu, \sigma_{i}^{2}+\sigma_{\mathrm{NLOS}}^{2}\right)$. According to the description in Figure 4 , the NLOS detection probability will occur in the regions $\left[-\infty, m-2.58 \sigma_{i}\right]$ and $\left[m+2.58 \sigma_{i},+\infty\right]$. Hence, $P_{\mathrm{D}}$ can be described as follows:

$$
P_{\mathrm{D}}=\Phi\left(\frac{m-2.58 \sigma_{i}-m-\mu}{\sqrt{\sigma_{i}^{2}+\sigma_{\mathrm{NLOS}}^{2}}}\right)+\left[1-\Phi\left(\frac{m+2.58 \sigma_{i}-m-\mu}{\sqrt{\sigma_{i}^{2}+\sigma_{\mathrm{NLOS}}^{2}}}\right)\right]
$$

Utilizing the simulated parameter value of measurement noise and NLOS distribution (see Section 4 ), $P_{\mathrm{D}}$ can be calculated as 0.64 .

Note that the two probabilities $P_{\mathrm{FA}}$ and $P_{\mathrm{D}}$ are correlated with the predefined threshold parameters. In the above-given parameters, $P_{\mathrm{D}}$ seems small. The reason is that the above predefined threshold parameters are based on the given minimum false alarm probability. If the given false alarm probability increases, the $P_{\mathrm{D}}$ will also increase.

\subsection{Maximum likelihood estimator}

Using each BS and the corresponding measurement condition, the joint probability density function of all the distance measurements at time instant $k$ can be readily obtained. After ignoring the constant, the log likelihood function of all the distance measurements can be written as [15]

$$
f\left(x_{k}, y_{k}\right)=\sum_{i=1}^{N} \frac{\left(z_{i, k}-\left(d_{i, k}+\tilde{\mu}\right)\right)^{2}}{\tilde{\sigma}_{i}^{2}}
$$

where it is assumed that the measurement noise and bias errors are mutually independent and

$$
\begin{gathered}
\tilde{\mu}=\left\{\begin{array}{ccc}
0 & \text { LOS } \\
\mu & \text { NLOS } & \text { (Gaussian) } \\
\lambda & \text { NLOS } & \text { Exponential) }
\end{array}\right. \\
\tilde{\sigma}_{i}^{2}=\left\{\begin{array}{cc}
\sigma_{i}^{2} & \text { LOS } \\
\sigma_{i}^{2}+\sigma_{\mathrm{NLOS}}^{2} & \text { NLOS (Gaussian) } \\
\sigma_{i}^{2}+\lambda^{2} & \text { NLOS (Exponential) }
\end{array}\right.
\end{gathered}
$$

Note that (1) NLOS statistics are assumed known in advance; (2) the sum of a Gaussian measurement noise and an exponentially distributed NLOS error may be approximated to have a Gaussian distribution with the mean equal to $\lambda$ and variance being $\sigma_{i}^{2}+\lambda^{2}$ for the scenarios where the NLOS bias is not the dominant error [5]. 
Applying the MLE produces the mobile position estimate as follows:

$$
\left(\hat{x}_{k}, \hat{y}_{k}\right)_{\mathrm{MLE}}=\underset{x_{k}, y_{k}}{\arg \min } f\left(x_{k}, y_{k}\right)
$$

Clearly, without distinguishing between the LOS and NLOS propagation, the MLE would be very sensitive to the NLOS effect to produce poor position estimation performance. Since the NLOS corrupted measurements can be identified with a large possibility by the possibility calculation, the NLOS effect can be significantly mitigated.

\subsection{Kalman filtering}

Once the intermediate position estimates are available, they can be filtered to produce better position estimates by a KF. The target motion state at time instant $k$ is defined as

$$
\mathbf{x}(k)=[x(k), y(k), \dot{x}(k), \dot{y}(k)]^{T}
$$

where $[x(k), y(k)]$ represents the position state of target and $[\dot{x}(k), \dot{y}(k)]$ denotes the corresponding velocity state. The target state with random acceleration can be modeled as $[22,24]$

$$
\mathbf{x}(k)=\mathbf{A x}(k-1)+\mathbf{w}(k-1)
$$

where the state transition matrix $\mathbf{A}=\left[\begin{array}{cc}\mathbf{I}_{2} & \Delta t \mathbf{I}_{2} \\ 0 & \mathbf{I}_{2}\end{array}\right]$ where $\mathbf{I}_{\mathbf{2}}$ is the $2 \times 2$ identity matrix and $\Delta t$ is the discrete time sampling interval. The random process $\mathbf{w}(k-1)$ is a zero-mean white Gaussian noise vector with covariance matrix

$$
\mathbf{Q}=\left[\begin{array}{cc}
\frac{\Delta t^{4}}{4} \mathbf{B} & \frac{\Delta t^{3}}{2} \mathbf{B} \\
\frac{\Delta t^{3}}{2} \mathbf{B} & \Delta t^{2} \mathbf{B}
\end{array}\right], \quad \mathbf{B}=\left[\begin{array}{cc}
\sigma_{x}^{2} & 0 \\
0 & \sigma_{y}^{2}
\end{array}\right]
$$

where $\sigma_{x}^{2}$ and $\sigma_{y}^{2}$ are the variances of the $x$-coordinate and $y$-coordinate acceleration noise component, respectively.

After applying the MLE, we can obtain the intermediate target position estimate $\overline{\mathbf{z}}(k)=[\bar{x}(k), \bar{y}(k)]^{T}$ and the estimation covariance matrix. The intermediate position estimate can be described as a linear equation of the target state:

$$
\overline{\mathbf{z}}(k)=\mathbf{H x}(k)+\mathbf{v}(k), \quad \mathbf{H}=\left[\begin{array}{llll}
1 & 0 & 0 & 0 \\
0 & 1 & 0 & 0
\end{array}\right]
$$

According to the Lemma 3.2 described in [11], the noise $\mathbf{v}(k)$ is white Gaussian with zero mean and covariance matrix $\mathbf{R}(k)$ given by

$$
\mathbf{R}(k)=\left[\begin{array}{ll}
\frac{\partial^{2} f(x, y)}{\partial x^{2}} & \frac{\partial^{2} f(x, y)}{\partial x \partial y} \\
\frac{\partial^{2} f(x, y)}{\partial y \partial x} & \frac{\partial^{2} f(x, y)}{\partial y^{2}}
\end{array}\right]_{x=\bar{x}_{k}, y=\bar{y}_{k}}^{-1}
$$

Thus, the prediction and update of the state and the covariance in implementing the standard KF are performed according to the following:

$$
\begin{aligned}
& \hat{\mathbf{x}}(k / k-1)=\mathbf{A} \hat{\mathbf{x}}(k-1 / k-1) \\
& \mathbf{P}(k / k-1)=\mathbf{A P}(k-1 / k-1) \mathbf{A}^{T}+\mathbf{Q}(k-1) \\
& \mathbf{K}(k)=\mathbf{P}(k / k-1) \mathbf{H}^{T}\left[\mathbf{R}(k)+\mathbf{H P}(k / k-1) \mathbf{H}^{T}\right]^{-1} \\
& \hat{\mathbf{x}}(k / k)=\hat{\mathbf{x}}(k / k-1)+\mathbf{K}(k)[\overline{\mathbf{z}}(k)-\mathbf{H} \hat{\mathbf{x}}(k / k-1)] \\
& \mathbf{P}(k / k)=[\mathbf{I}-\mathbf{K}(k) \mathbf{H}] \mathbf{P}(k / k-1)
\end{aligned}
$$

where $\mathbf{K}(k)$ is the Kalman gain, $\hat{\mathbf{x}}(k / k)$ is the state update including the desired position estimate at time instant $k$, and $\mathbf{P}(k / k-1)$ and $\mathbf{P}(k / k)$ are the state covariance prediction and the state covariance update, respectively.

\subsection{Summary}

The proposed approach can be described by the flow chart shown in Figure 5, consisting of the following steps.

1. Obtain the measurement prediction of each BS using Kalman position prediction, then apply the truncated triangular probability-possibility transformation to calculate the possibility of each measurement.

2. Select $N_{\mathrm{s}}$ measurements which have the high possibilities as LOS measurements and the other $\left(N-N_{\mathrm{s}}\right)$ measurements which have the low possibilities as NLOS measurements for single-shot position determination, according to the predefined numbers of LOS measurement or the predefined possibility threshold.

3. Use the Kalman position prediction as the initial position estimate and then estimate the target position with MLE; meanwhile, initialize the covariance matrix for Kalman filtering.

4. Use the standard KF to filter the MLE-based single-shot position estimates to produce the final position estimates of the target; meanwhile, feedback Kalman position prediction to MLE and make measurement prediction for fuzzy modeling. 


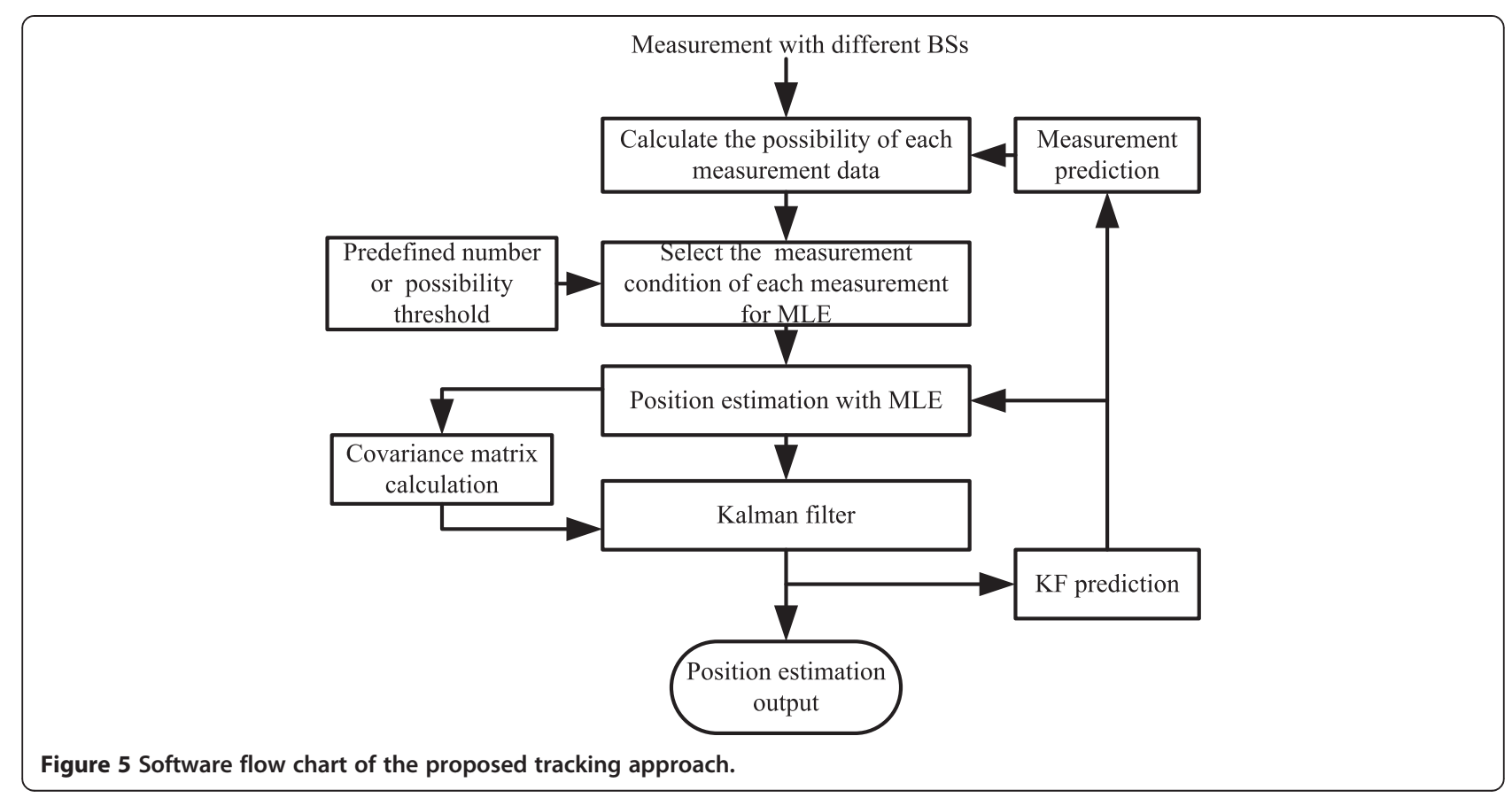

\section{Simulation results}

In this section, simulation results are provided to assess the performance of the proposed three-step tracking approach. It is assumed that the target can communicate with seven BSs, whose position coordinates (in meters) are set at $(6,000,0),(3,000,-6,000),(-3,000,-5,000)$, $(-6,000,-1,000),(-4,000,6,000),(0,5,000)$ and $(4,000$, $6,000)$ respectively. The noise $n_{i, k}$ and the bias error $e_{i, k}$ are assumed Gaussian with $\sigma_{i}=150 m$ (for $i=1, \ldots 7$ ), $\sigma_{\mathrm{NLOS}}=409 \mathrm{~m}$ and $\mu=513 \mathrm{~m}$. These parameters are similar to those selected in $[7,15]$. In random NLOS conditions, the channel propagation alternates between LOS and NLOS in a random pattern. Three different NLOS occurrence rates are considered, which are $10 \%$, $30 \%$, and $50 \%$, respectively. Meanwhile, in the fixed NLOS condition, only the measurements at the $\mathrm{BS}_{2}$ and $\mathrm{BS}_{5}$ contain the NLOS error all the time, whereas the measurements of other BSs are assumed under LOS condition. For simplicity, the trajectory of the mobile for simulation is shown in Figure 6. The mobile travels

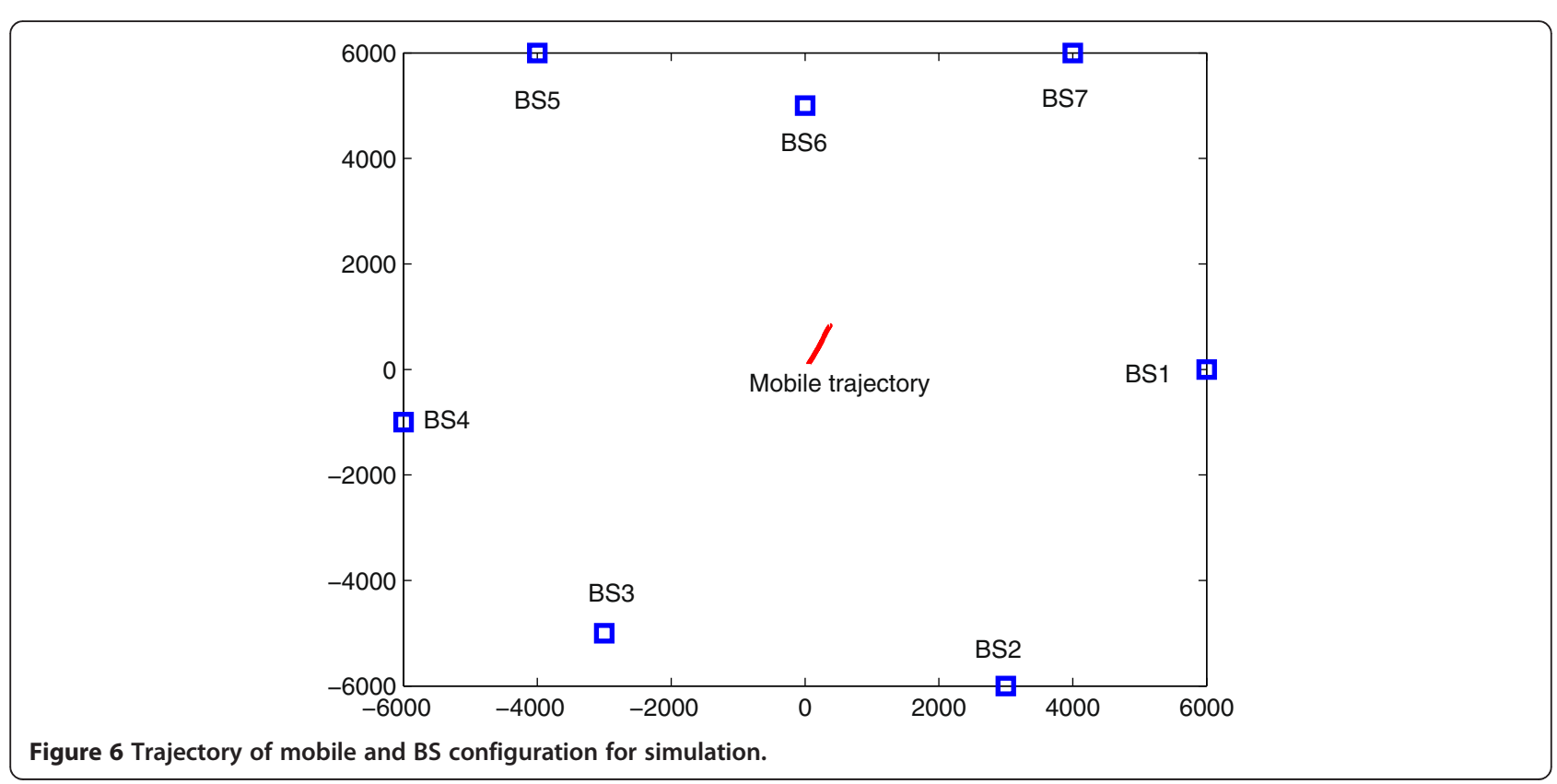


from point $(50 \mathrm{~m}, 100 \mathrm{~m})$ at a velocity of $v_{x}=5 \mathrm{~m} / \mathrm{s}$, $v_{y}=10 \mathrm{~m} / \mathrm{s}$. The random acceleration variances $\sigma_{x}^{2}$ and $\sigma_{y}^{2}$ are both chosen to be $0.5\left(\mathrm{~m} / \mathrm{s}^{2}\right)$.

The sampling interval is $\Delta t=0.05 \mathrm{~s}$ and there are 1,500 distance measurements associated with each base station for each simulation. A simple linear least squares method is utilized to generate the initial position value [25-27]. The initial velocity state is set at zero. The initial covariance matrix is set as $I_{4} \times{ }_{4}$ multiplied by 1,000 . The predefined number for LOS measurement (i.e., the number of BSs) is denoted by $N_{\mathrm{s}}$.

Figure 7 shows the average of root mean square error (RMSE) of the proposed approach with respect to the number of selected BSs $\left(N_{\mathrm{s}}\right)$ in random NLOS environments. The RMSE of the position estimates is defined by

$\operatorname{RMSE}=\sqrt{\frac{1}{M} \sum_{j=1}^{M}\left[\left(\hat{x}_{j}(k / k)-x(k)\right)^{2}+\left(\hat{y}_{j}(k / k)-y(k)\right)^{2}\right]}$

where $\left[\hat{x}_{j}(k / k), \hat{y}_{j}(k / k)\right]$ is the corresponding position estimation at the $k$ th time instant in the $j$ th Monte Carlo run and $M$ is the total number of Monte Carlo test.

It can be observed that the performance can be improved as the predefined number increases when the NLOS occurrence rate is $10 \%$. However, the outcome is different in the scenario of $30 \%$ and $50 \%$ NLOS occurrence rate. This phenomenon can be associated with the channel propagation conditions. In the case of a large NLOS occurrence rate, as the predefined number of selected measurements increases, more NLOScorrupted measurements would be selected as LOS measurements, resulting in performance degradation.
Thus, for the predefined number-based approach, some prior knowledge about the channel condition should be known in advance if we want to obtain better location performance. In order to overcome this shortcoming, a method based on predefined possibility threshold is investigated next for the measurement selection.

Figure 8 shows the performance in terms of average RMSE with respect to different predefined possibility threshold. It can be seen that the average RMSE is smallest in all four simulation scenarios, when the threshold is equal to 0.12 . Therefore, for practical application, the appropriate threshold should be set at 0.12 . Table 2 shows the average number of selected LOS BSs used for position determination. For instance, when the threshold is selected as 0.12 , in random NLOS (30\%) condition, for each BS, the false alarm probability is equal to $(1-0.3) \times 0.01=0.007$, and the error probability which considers the measurement as LOS measurement in NLOS scenario can be calculated as $0.3 \times(1-0.64)=$ 0.108 . Hence, the average number of LOS BSs is obtained as $7 \times[(1-0.3)-0.007+0.108]=5.607$. Compared with the simulation result, it obviously agrees with the theory analysis result in this environment.

According to the discussion above, predefined possibility threshold-based method is utilized for the following simulation comparisons when the threshold equals 0.12 . Figure 9 shows the RMSE comparison between the proposed BS selection and idealized cases. For idealized cases, the LOS/NLOS state of each BS in the trajectory is known in advance. From the figure, it can be seen that the performance of the proposed approach is close to that of idealized cases. These results also reflect the

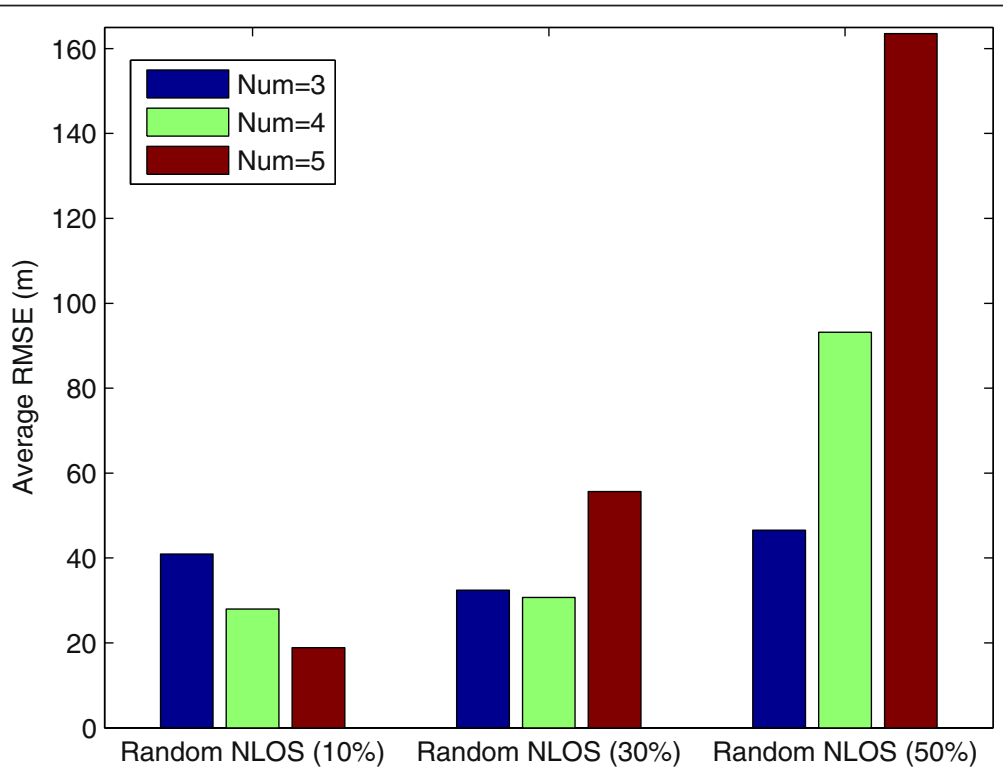

Figure 7 Average RMSE versus number of selected BSs and random NLOS percentage. 


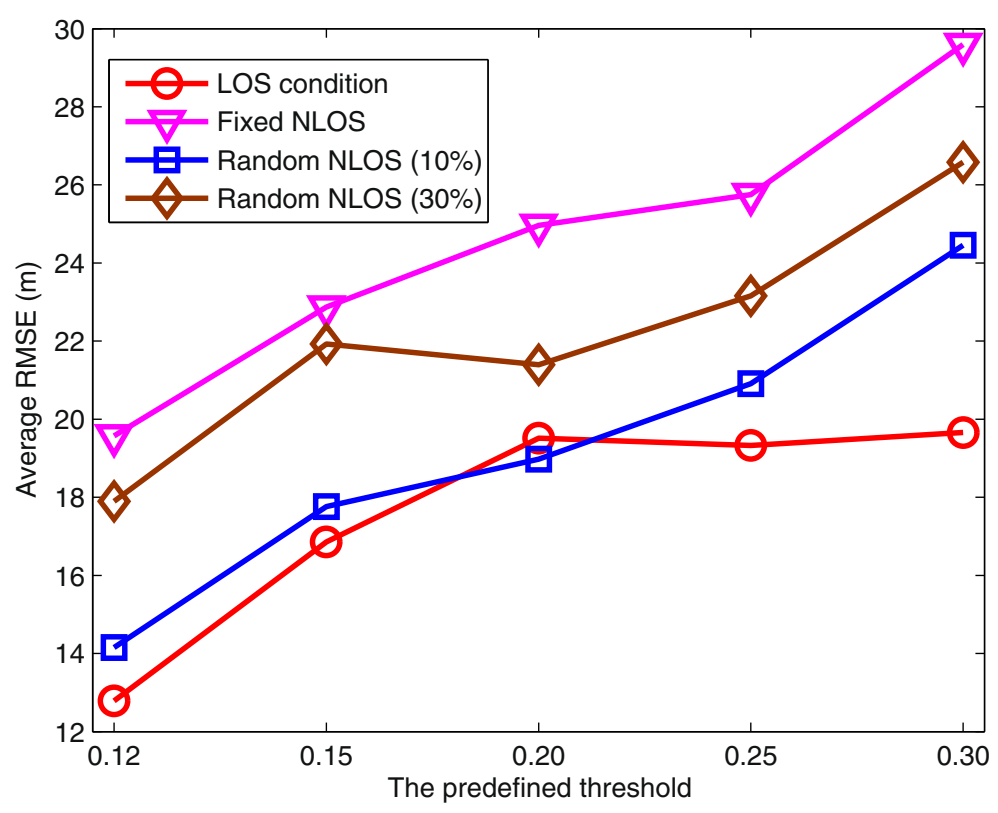

Figure 8 Average RMSE versus the predefined possibility threshold.

effect of the fuzzy modeling for LOS/NLOS identification. As the NLOS occurrence rate becomes large, the RMSE increases because it is more likely that one or more NLOS corrupted measurements would be selected as LOS measurement. However, the performance difference between the $10 \%$ and $30 \%$ cases is not significant. The average of the RMSE of the idealized case at the initial position is 25.3 and $25.8 \mathrm{~m}$ for the $10 \%$ and $30 \%$ NLOS, respectively. Also, the average of the RMSE of the proposed method at the corresponding position is 30.5 and $31.5 \mathrm{~m}$ for the $10 \%$ and $30 \%$ NLOS, respectively. The reason may be contributed to the NLOS mitigation method in the proposed algorithm. Figure 10 illustrates the performance comparison between different random acceleration variances when the NLOS occurrence rate is set at $30 \%$. As expected, when the acceleration variance increases, the RMSE becomes larger. However, the performance degradation is not dramatic, although the variance increases by $400 \%$. Therefore, the performance is relatively insensitive to the selection of the acceleration noise variance.

Table 2 Average number of selected base stations

\begin{tabular}{llllll}
\hline Channel condition & \multicolumn{5}{c}{ Predefined possibility threshold } \\
\cline { 2 - 6 } & $\mathbf{0 . 1 2}$ & $\mathbf{0 . 1 5}$ & $\mathbf{0 . 2 0}$ & $\mathbf{0 . 2 5}$ & $\mathbf{0 . 3 0}$ \\
\hline LOS condition & 6.924 & 6.032 & 5.850 & 5.665 & 5.430 \\
Fixed NLOS & 5.682 & 4.723 & 4.564 & 4.393 & 4.214 \\
Random NLOS (10\%) & 6.486 & 5.567 & 5.413 & 5.216 & 4.998 \\
Random NLOS (30\%) & 5.623 & 4.654 & 4.495 & 4.345 & 4.153 \\
\hline
\end{tabular}

Let us examine the reliability of the probability calculated based on the proposed approach. Figure 11 shows the simulated LOS probabilities of all seven BSs in four different propagation environments. Taking 30\% NLOS occurrence rate as an example, from the discussion in subsection 3.1, the false alarm probability in given condition is $P_{\mathrm{FA}} \times(1-0.3)=0.007$ and the corresponding error probability can be calculated as $\left(1-P_{\mathrm{D}}\right) \times 0.3=$ 0.108 . Since the NLOS rate is 0.3 , the LOS probability is 0.7 and thus the actual calculated LOS probability of the proposed algorithm is $0.7+0.108-0.007=0.801$. In addition, in the fixed NLOS condition, the false alarm probabilities in LOS and NLOS conditions are $P_{\mathrm{FA}} \times$ $(1-0)=0.01$ and $P_{\mathrm{FA}} \times(1-1)=0$, respectively. The corresponding error probabilities can also be calculated as $\left(1-P_{\mathrm{D}}\right) \times 0=0$ (for LOS condition) and $\left(1-P_{\mathrm{D}}\right) \times 1=0.36$ (for NLOS condition). The actual calculated LOS probabilities are $1-0.01+0=0.99$ for LOS condition and $0-$ $0+0.36=0.36$ for NLOS condition. From Figure 11, it can be found that the simulation results are in good agreement with the theoretical analysis. Hence, the calculated possibility can be utilized to accurately identify the LOS/NLOS scenarios.

Then, we compare the performance of the proposed approach with that of the algorithms proposed in $[11,15]$. In the simulation, the prior knowledge of LOS and NLOS for each BS is known in advance for the algorithm in [11]. Hence, the simulated results also can be considered as the results obtained from the idealized case. For the BS selection method in [15], the predefined number of selected $\mathrm{BS}$ and the measurement 

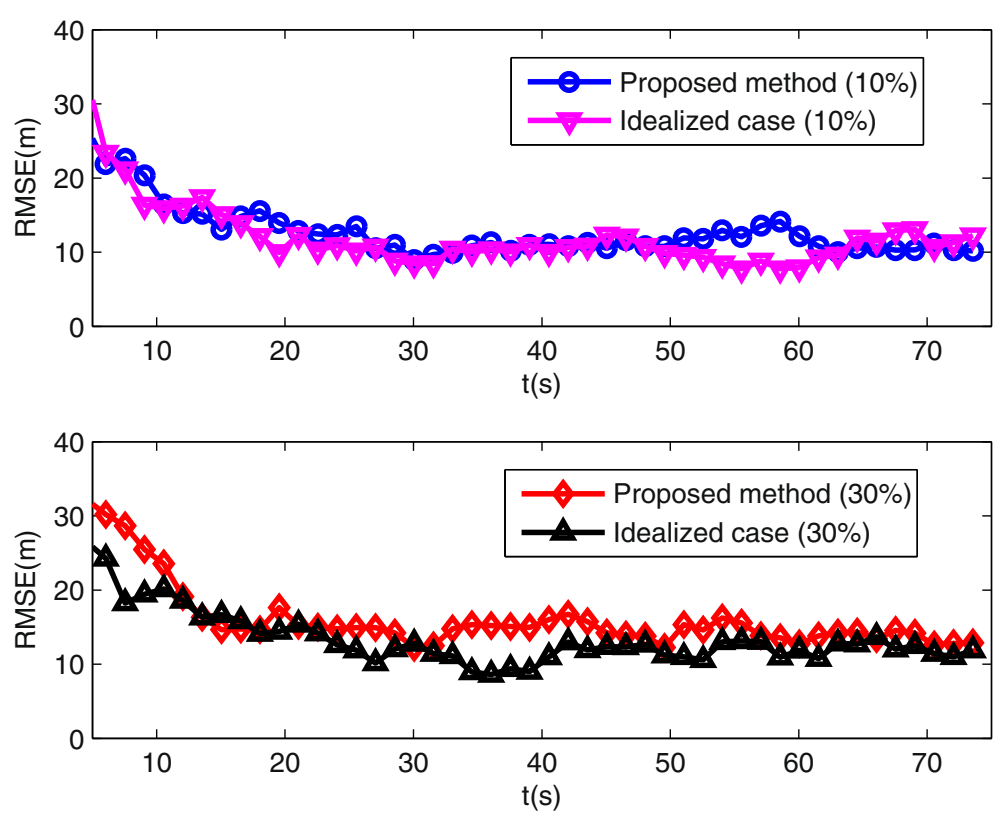

Figure 9 RMSE comparison with the idealized cases.

records are 3 and 20. The step of position estimation is the same as the proposed algorithm. Figures 12, 13, 14, 15 describe the cumulative distribution function (CDF) of the position error of different algorithms in different scenarios. From the simulation results, it can be seen that on average, the proposed method significantly outperforms the method in [15] and is close to that of the idealized case. Because the method of [15] only selects the best TOA measurements for position estimation, it will lose some performance gain when the NLOS measurements and some LOS measurements are not utilized. Meanwhile, proper parameter initialization is also crucial to obtain better position estimation. From the figures, it can be seen that the performance of [15] varies in different environments for the given parameters. In this sense, it also requires proper prior knowledge

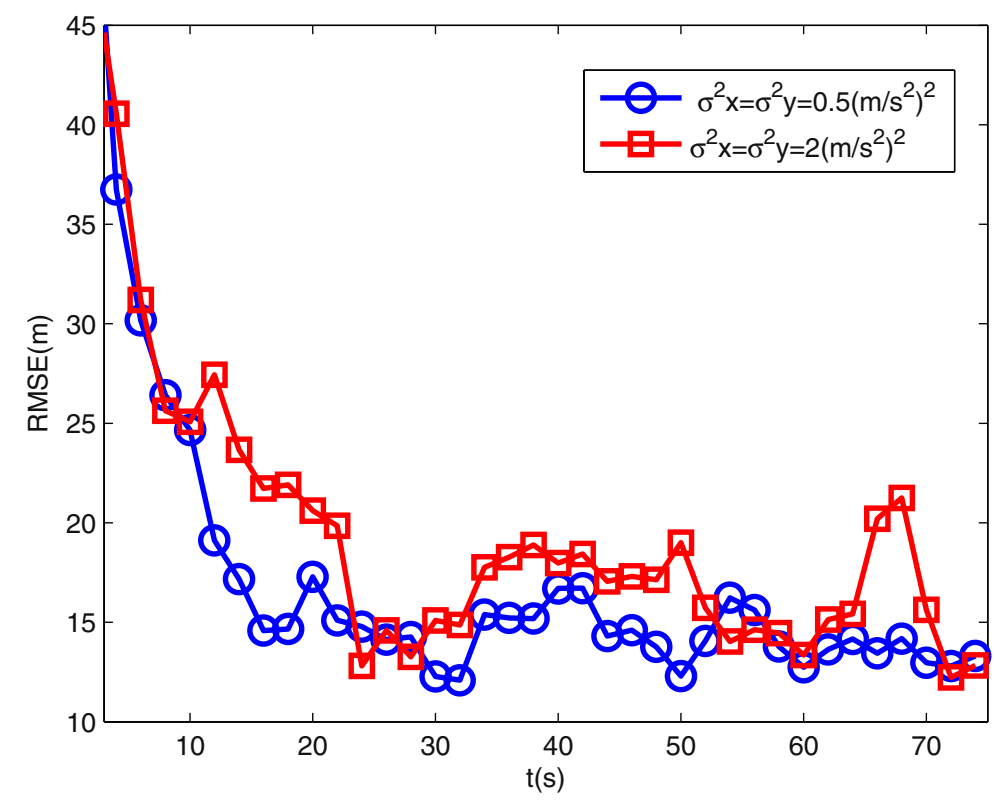

Figure 10 RMSE versus the random acceleration variances. 


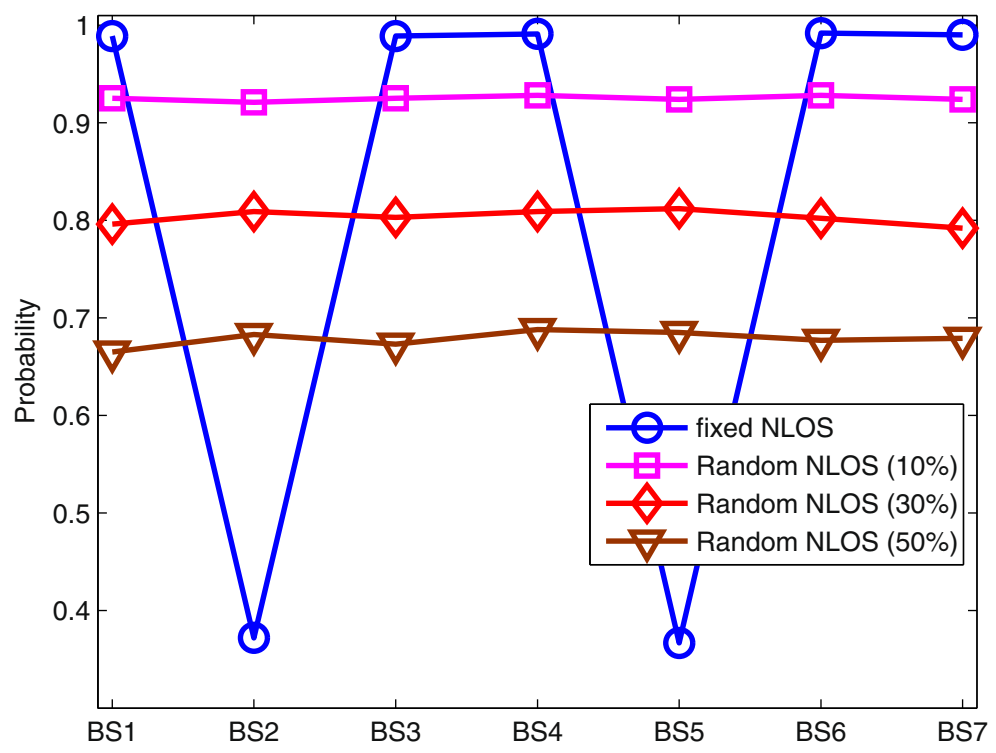

Figure 11 The LOS probabilities of each BS.

of the channel environments. On the other hand, although the performance of [11] is better than that of the proposed algorithm, it should be noted that these acquired results require accurate prior knowledge of the environments. For practical application, the method of [11] should add the LOS/NLOS identification function so that both MLE and covariance calculation can be realized. As a consequence, the method in [11] would become much more complicated. In the proposed approach, the position estimation performance relies on the accuracy of the proposed LOS/NLOS identification method. However, the proposed algorithm does not require any additional step for position estimation and the performance is close to that of the idealized case. Besides, the LOS/ NLOS selection results with $30 \%$ NLOS condition are illustrated in Figure 16. For clarity, only the results over the time interval between 800 and 900 are shown. From Figure 16, it can be seen that most of NLOS condition can be identified by the proposed algorithm. The missed and incorrect identifications are associated with the loss and alarm probability of the proposed approach.

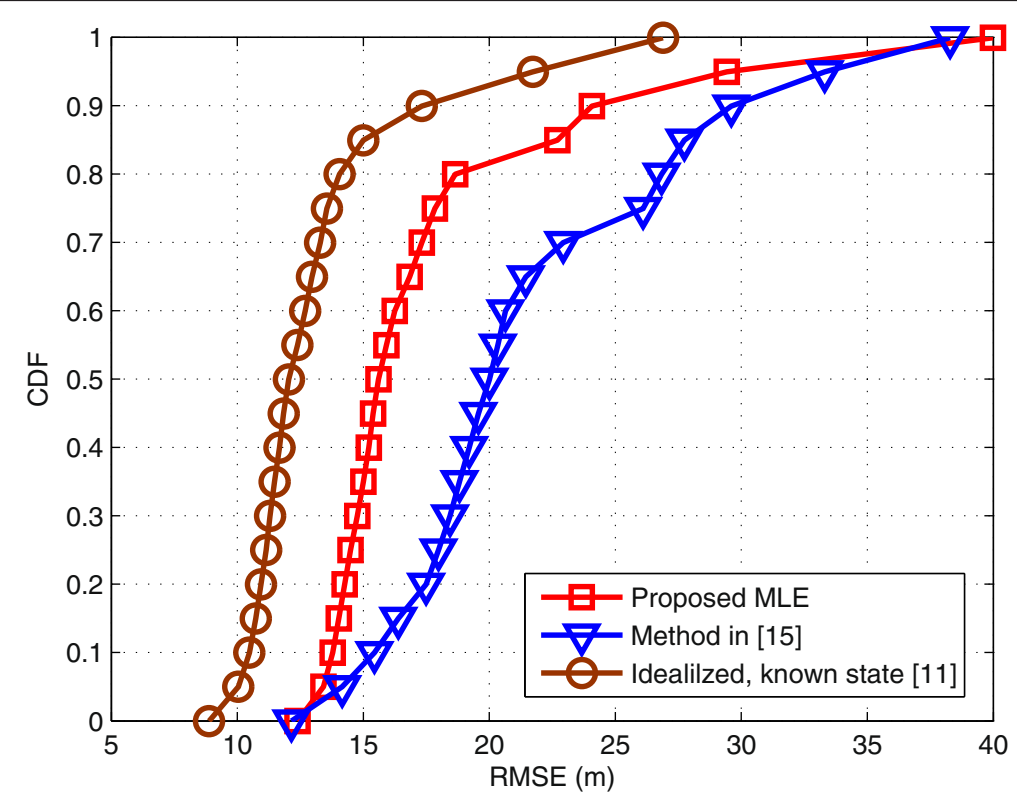

Figure 12 Algorithm comparison in fixed NLOS condition. 


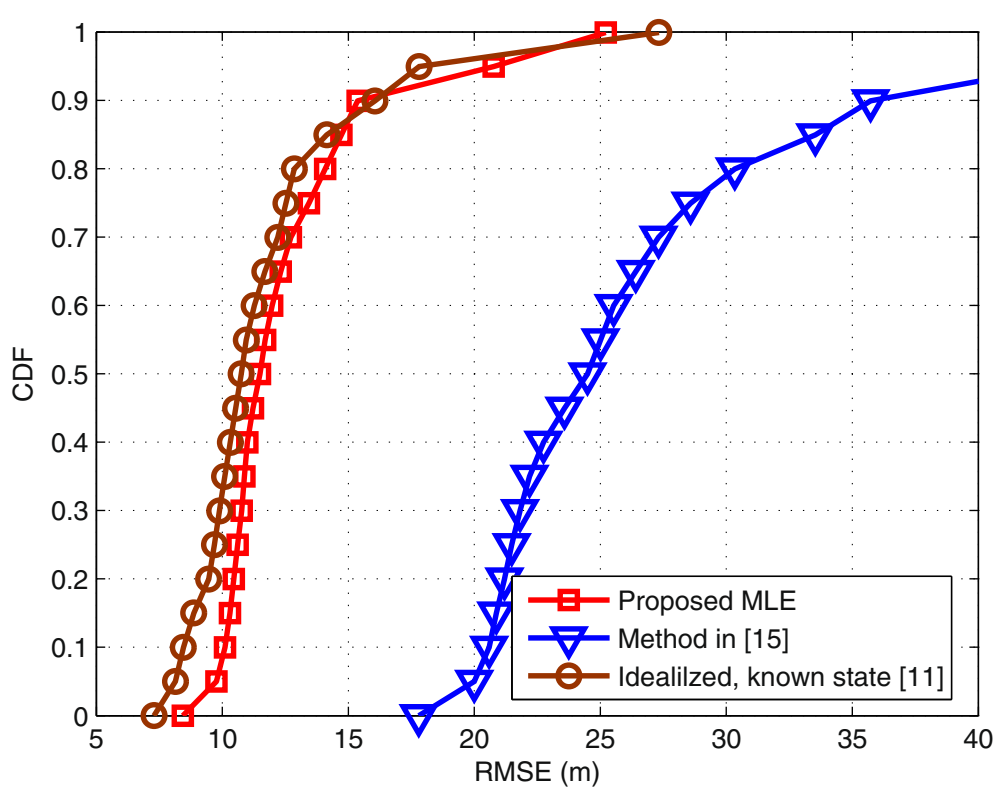

Figure 13 Algorithm comparison in $10 \%$ NLOS occurrence rate.

Next, some simulations are designed for the case where the NLOS error is assumed to be an exponential variable $(\lambda=300 \mathrm{~m})$ and the measurement noise is Gaussian with mean zero and STD $\sigma_{i}=30 \mathrm{~m}$ [28]. Figure 17 shows the corresponding $\mathrm{CDF}$ of the simulated position error. From the simulation results, it can be seen that although the NLOS error does not follow the Gaussian distribution, the proposed algorithm also performs well. Thus, the proposed algorithm is suited for different NLOS distributions. Figure 18 shows the performance comparison among three different algorithms for the case where the NLOS occurrence rate is $30 \%$ and the NLOS error has an exponential distribution [28]. It can be seen that the conclusions with Gaussian distribution can also be obtained.

At last, the performance analysis of the proposed algorithm is described, when the prior NLOS statistics are incorrect. Taking the $30 \%$ NLOS occurrence rate as an example, the mean of NLOS bias is set to be $200 \mathrm{~m}$, but it is assumed to be $513 \mathrm{~m}$ incorrectly. From Figure 19, it

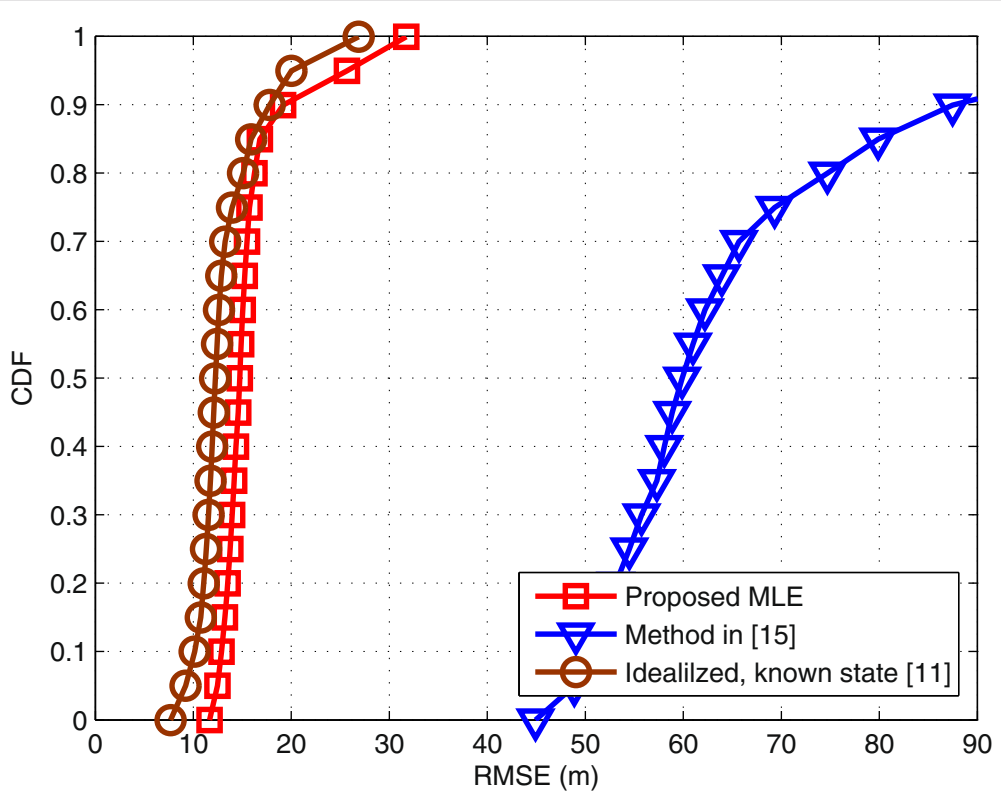

Figure 14 Algorithm comparison in $30 \%$ NLOS occurrence rate. 


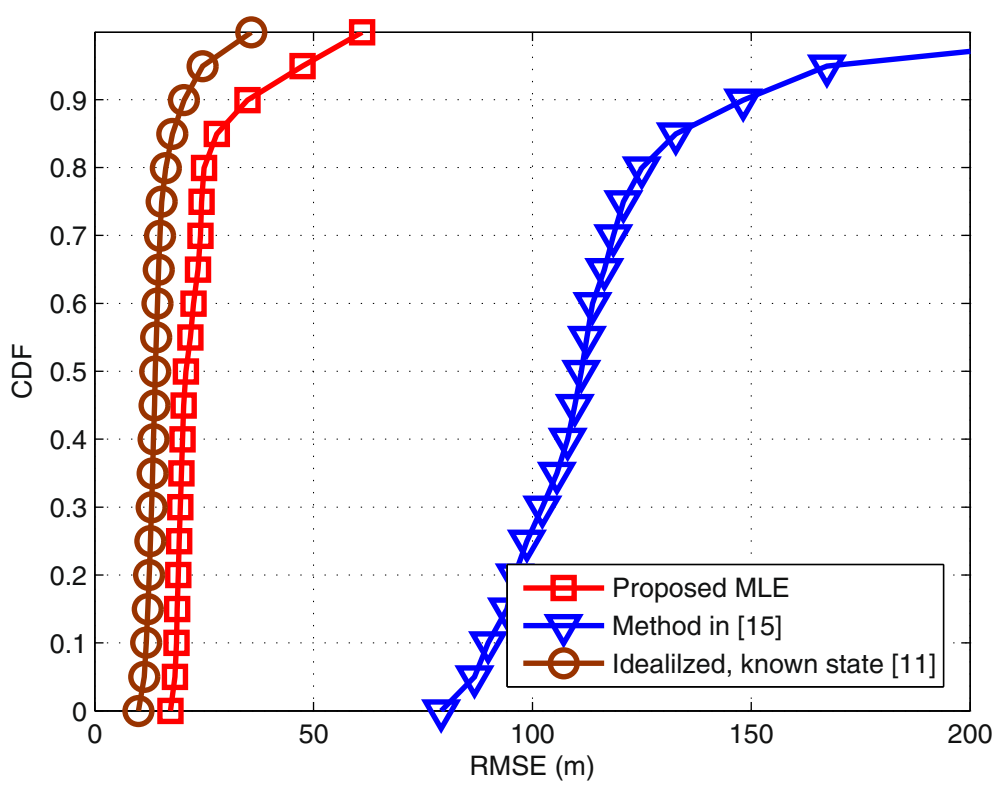

Figure 15 Algorithm comparison in 50\% NLOS occurrence rate.

can be seen that the performance with the incorrect NLOS statistics is similar to that with correct statistics.

\section{Conclusions}

In this article, a three-step positioning algorithm is proposed to mitigate NLOS propagation effect and to achieve desirable positioning accuracy for target tracking in NLOS propagation environments. A fuzzy modeling method is developed to identify whether the distance measurements are under LOS condition and the measurements are employed for MLE-based position determination, according to the identified measurement condition. The intermediate position estimates are smoothed using a linear KF to produce better positioning performance. The position prediction from the KF is utilized for parameter initialization in the MLE and for fuzzy modeling-based measurement selection. In the case where there are measurements corrupted by NLOS propagation and the LOS and NLOS conditions are unknown, the proposed approach outperforms the existing algorithms.

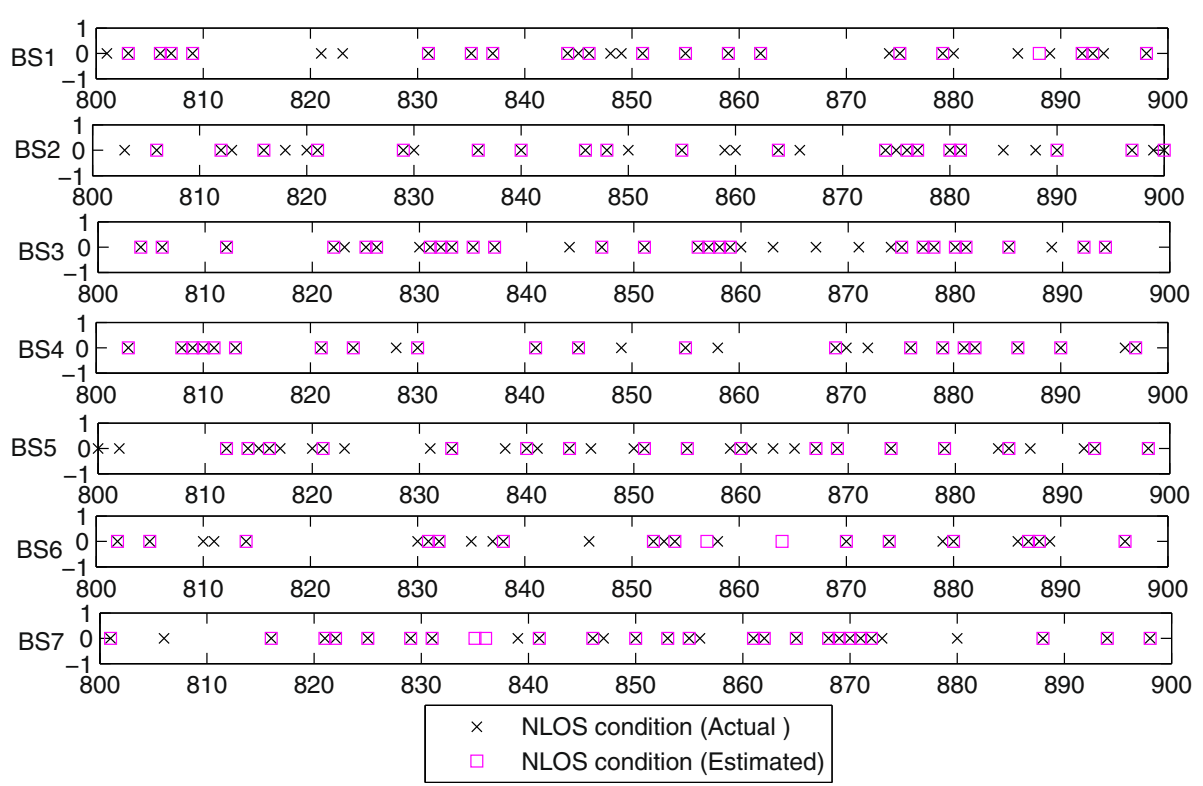

Figure 16 The description of actual and estimated NLOS condition. 


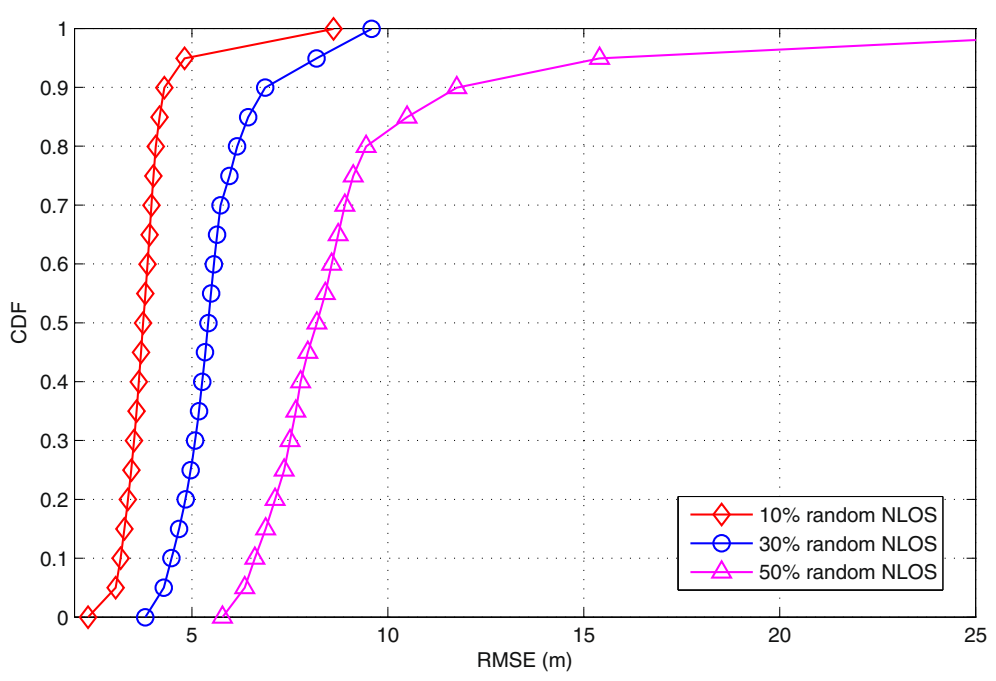

Figure 17 CDFs in random NLOS scenarios.

\section{Appendix}

The parameter selection criterion is based on the NP detection theory [29]. In this paper, the prior given false alarm probability is chosen as minimum false alarm probability.

First, a two-hypothesis testing is defined:

$$
\left\{\begin{array}{ccc}
H_{0}: & z_{i k}=d_{i k}+n_{i k} & \text { LOS } \\
H_{1}: & z_{i k}=d_{i k}+n_{i k}+e_{i k} & \text { NLOS }
\end{array}\right.
$$

The mean of $d_{i k}$ is the actual distance between the $i$ th base station and the MS at time instant $k$. In this paper, Equation 4 can be approximated to describe this actual distance. Hence, as seen in Figure 3, the parameters for probability-possibility transformation can be defined as follows:

$$
x_{c}=m, x_{\varepsilon}=m+1.54 \sigma_{i}, x_{n}=m+2.58 \sigma_{i}
$$

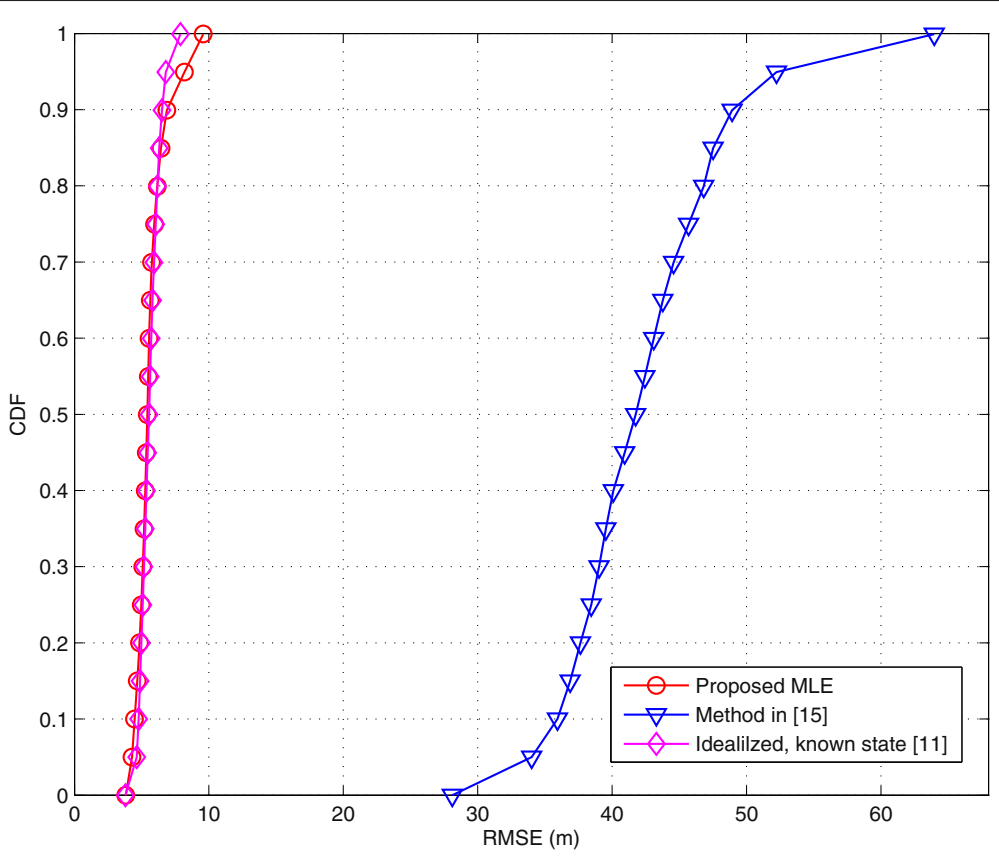

Figure 18 Algorithm comparison with exponential distributed NLOS. 


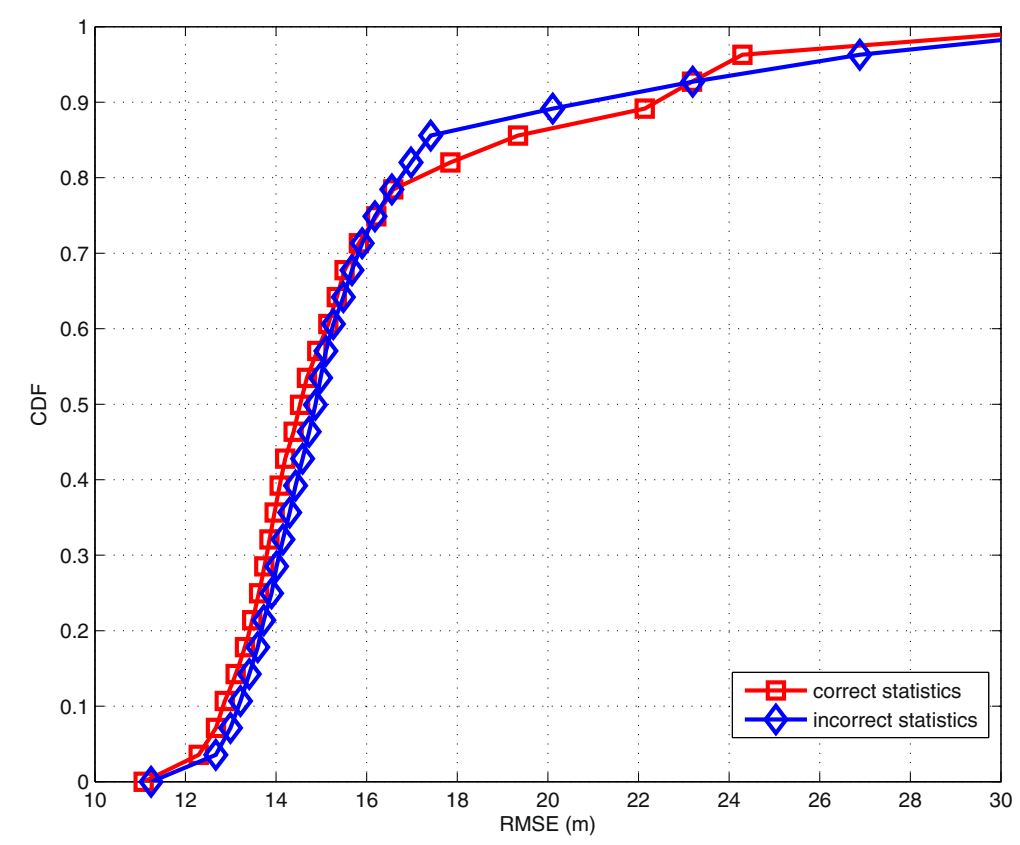

Figure 19 Performance analysis with incorrect NLOS statistics.

Through the fuzzy modeling processing, the test can be described as follows:

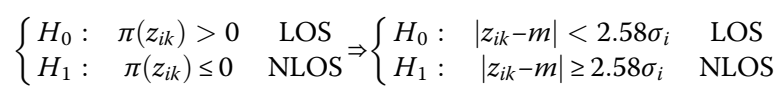

With the knowledge of NP detection theory, in the following, the prior given false alarm probability is chosen as the minimum false alarm probability and then to calculate the detection probability in this given condition. Furthermore, we also judge whether the obtained detection probability is the maximum.

First, we will describe how to obtain the parameters in the minimum false alarm probability condition. From (20), it can be seen that in LOS condition, $z_{i k} \sim N\left(m, \sigma_{i}^{2}\right)$. Assume that the left and right endpoints of the predefined parameters in this condition are $m-A_{1} \sigma_{i}$ and $m+$ $A_{2} \sigma_{i}$, respectively. From (21), it can also be seen that $A_{1} \leq 2.58, A_{2} \leq 2.58$. Then, the false alarm probability $P_{\mathrm{FA}}$ can be described as follows:

$$
\begin{aligned}
P_{\mathrm{FA}}= & P\left(H_{1} ; H_{0}\right)=\Phi\left(\frac{m-A_{1} \sigma_{i}-m}{\sigma_{i}}\right) \\
& +\left(1-\Phi\left(\frac{m+A_{2} \sigma_{i}-m}{\sigma_{i}}\right)\right)=2-\Phi\left(A_{1}\right)-\Phi\left(A_{2}\right)
\end{aligned}
$$

Since $\Phi(x)$ is a monotone increasing function, in order to make $P_{\mathrm{FA}}$ minimum, $A_{1}$ and $A_{2}$ must be maximum. Therefore, the predefined two endpoints to achieve minimum $P_{\mathrm{FA}}$ are $m-2.58 \sigma_{i}$ and $m+2.58 \sigma_{i}$, respectively. According to the probability-possibility transformation described in Figure 3, we can also obtain the predefined possibility parameter $\varepsilon=0.12$.

Second, we will validate whether the detection probability is maximum with the above obtained parameters.

From (20), it can be seen that in NLOS condition, $z_{i k} \sim$ $N\left(m+\mu, \sigma_{i}^{2}+\sigma_{\mathrm{NLOS}}^{2}\right)$. Assume that the left and right endpoints of the predefined parameters are $m-A_{3} \sigma_{i}$ and $m+A_{4} \sigma_{i}$, respectively. From (21), it can also be obtained that $A_{3} \geq 2.58, A_{4} \geq 2.58$. Then, the detection probability $P_{\mathrm{D}}$ can be described as follows:

$$
\begin{aligned}
P_{\mathrm{D}}= & \Phi\left(\frac{m-A_{3} \sigma_{i}-m-\mu}{\sqrt{\sigma_{i}^{2}+\sigma_{\mathrm{NLOS}}^{2}}}\right) \\
& +\left[1-\Phi\left(\frac{m+A_{4} \sigma_{i}-m-\mu}{\sqrt{\sigma_{i}^{2}+\sigma_{\mathrm{NLOS}}^{2}}}\right)\right] \\
= & 2-\Phi\left(\frac{A_{4} \sigma_{i}-\mu}{\sqrt{\sigma_{i}^{2}+\sigma_{\mathrm{NLOS}}^{2}}}\right)-\Phi\left(\frac{A_{3} \sigma_{i}+\mu}{\sqrt{\sigma_{i}^{2}+\sigma_{\mathrm{NLOS}}^{2}}}\right)
\end{aligned}
$$

It can be seen that is $P_{\mathrm{D}}$ a monotone decreasing function with parameters of $A_{3}, A_{4}$. Hence, in order to make $P_{\mathrm{D}}$ maximum, the parameters are also $m-2.58 \sigma_{i}$, $m+2.58 \sigma_{i}$. Similarly, with the probability-possibility transformation, the predefined possibility parameter is chosen as $\varepsilon=0.12$. 
From the discussion above, it can be concluded that for a given $P_{\mathrm{FA}}$, the proposed NLOS identification test can make $P_{\mathrm{D}}$ maximum. This test agrees with NP detection theory.

\section{Competing interests}

The authors declare that they have no competing interests.

\section{Acknowledgements}

The work was supported by the National Natural Science Foundation of China (Nos. 61302103,61372122, 61372123, 61174205, and 61170252) and the Research Foundation for Advanced Talents of NJUPT (NY213012).

\section{Author details}

${ }^{1}$ College of Telecommunications and Information Engineering, Nanjing University of Posts and Telecommunications, Nanjing 210003, China. ${ }^{2}$ School of Geodesy and Geomatics, Wuhan University, Wuhan 430072, China. ${ }^{3}$ School of Information Science and Engineering, Southeast University, Nanjing 210096, China.

\section{Received: 6 December 2013 Accepted: 16 June 2014} Published: 10 July 2014

\section{References}

1. K Yu, I Sharp, YJ Guo, Ground-Based Wireless Positioning (Wiley-lEEE, Hoboken, 2009)

2. S Gezici, HV Poor, Position estimation via ultra-wide-band signals. Proc. IEEE 97(2), 386-403 (2009)

3. S Ahonen, P Eskelinen, Mobile terminal location for UMTS. IEEE Aerosp. Electron. Syst. Mag. 18(2), 23-27 (2003)

4. M Anisetti, CA Ardagna, V Bellandi, E Damiani, Map-based location and tracking in multipath outdoor mobile networks. IEEE Trans. Wireless Commun. 10(3), 814-824 (2011)

5. K Yu, YJ Guo, Improved positioning algorithm for nonline-of-sight environments. IEEE Trans. Vehicular Technol. 57(4), 2342-2353 (2008)

6. W Kim, JG Lee, G-I Jee, The interior-point method for an optimal treatment of bias in trilateration location. IEEE Trans. Vehicular Technol. 55(4), 1291-1301 (2006)

7. H Miao, K Yu, M Juntti, Positioning for NLOS propagation: algorithm derivations and Cramer-Rao bounds. IEEE Trans. Vehicular Technol. 56, 2568-2580 (2007)

8. X Wang, Z Wang, B ODea, A TOA-based location algorithm reducing the errors due to non-line-of-sight (NLOS) propagation. IEEE Trans. Vehicular Technol. 52(1), 112-116 (2003)

9. K Yu, E Dutkiewicz, NLOS identification and mitigation for mobile tracking. IEEE Trans. Aerosp. Electron. Syst. 49(3), 1438-1452 (2013)

10. L Cong, W Zhuang, Nonline-of-sight error mitigation in mobile location. IEEE Trans. Wireless Commun. 4, 560-573 (2005)

11. X Wang, M Fu, H Zhang, Target tracking in wireless sensor networks based on the combination of KF and MLE using distance measurements. IEEE Trans. Mobile Comput. 11(4), 567-576 (2012)

12. BL Le, K Ahmed, H Tsuji, Mobile location estimator with NLOS mitigation using Kalman filtering, in Proceedings of Wireless Communications and Networking Conference. New Orleans, 16-20 March 2003, pp. 1969-1973

13. ZR Zaidi, BL Mark, Real-time mobility tracking algorithms for cellular networks based on Kalman filtering. IEEE Trans. Mobile Comput. 4(2), 195-208 (2005)

14. K Yu, E Dutkiewicz, Geometry and motion based positioning algorithms for mobile tracking in NLOS environments. IEEE Trans. Mobile Comput. 11(2), 254-263 (2012)

15. S Mazuelas, FA Lago, P Fernandez, A Bahillo, J Blas, RM Lorenzo, EJ Abril, Ranking of TOA measurements based on the estimate of the NLOS propagation contribution in a wireless location system. Wireless Pers. Commun. 53(1), 35-52 (2010)

16. JF Liao, BS Chen, Robust mobile location estimator with NLOS mitigation using interacting multiple model algorithm. IEEE Trans. Wireless Commun. 5(11), 3002-3006 (2006)

17. C Morelli, M Nicoli, V Rampa, U Spagnolini, Hidden Markov models for radio localization in mixed LOS/NLOS conditions. IEEE Trans. Signal Process. 55(4), 1525-1542 (2007)
18. U Hammes, AM Zoubir, Robust MT tracking based on M-estimation and interacting multiple model algorithm. IEEE Trans. Signal Process. 59(7), 3398-3409 (2011)

19. M Mcguire, KN Plataniotis, Dynamic model-based filtering for mobile terminal location estimation. IEEE Trans. Vehicular Technol. 52(4), 1012-1031 (2003)

20. JM Huerta, J Vidal, A Giremus, J-Y Tourneret, Joint particle filter and UKF position tracking in severe non-line-of-sight situations. IEEE J. Selected Topics Signal Process 3(5), 874-888 (2009)

21. G Mauris, V Lasserre, L Foulloy, Fuzzy modeling of measurement data acquired from physical sensors. IEEE Trans. Instrum. Meas. 49(6), 1201-1205 (2000)

22. L Chen, S Ali-Loytty, R Piche, LN Wu, Mobile tracking in mixed line-of-sight/ non-line-of-sight conditions: algorithm and theoretical lower bound. Wireless Pers. Commun. 65, 753-771 (2012)

23. L Chen, LN Wu, Mobile positioning in mixed LOS/NLOS conditions using modified EKF banks and data fusion method. IEICE Trans. Commun. E92-B(4), 1318-1325 (2009)

24. Y Bar-Shalom, XR Li, T Kirubarajan, Estimation with Applications to Tracking and Navigation, Theory Algorithms and Software (Wiley, New York, 2001)

25. Ml Silventoinen, T Rantalainen, Mobile station emergency locating in GSM, in IEEE International Conference on Personal Wireless Communications. India, 1996, pp. 232-238

26. JJ Caffery, A new approach to the geometry of TOA location, in the 52th IEEE Vehicular Technology Conference (VTC-Fall). Boston, MA, 24-28 September 2000, vol. 4, pp. 1943-1949

27. I Guvenc, S Gezici, F Watanabe, H Inamura, Enhancements to linear least squares localization through reference selection and ML estimation, in IEEE Wireless Communications and Networking Conference (WCNC). Las Vegas, 31 March to 3 April 2008, pp. 284-289

28. PC Chen, A non-line-of-sight error mitigation algorithm in location estimation, in IEEE Wireless Communications and Networking Conference (WCNC). New Orleans, 21-24 September 1999, vol. 1, pp. 316-320

29. SM Kay, Fundamentals of Statistical Signal Processing Volume II: Detection Theory (Prentice Hall, New Jersey, 1998)

doi:10.1186/1687-6180-2014-105

Cite this article as: Yan et al:: Fuzzy modeling, maximum likelihood estimation, and Kalman filtering for target tracking in NLOS scenarios. EURASIP Journal on Advances in Signal Processing 2014 2014:105.

\section{Submit your manuscript to a SpringerOpen ${ }^{\odot}$ journal and benefit from:}

- Convenient online submission

Rigorous peer review

- Immediate publication on acceptance

- Open access: articles freely available online

- High visibility within the field

- Retaining the copyright to your article

Submit your next manuscript at $>$ springeropen.com 